\title{
Case Report of Multinodular Goiter and Airway Compression in a Preeclamptic Patient
}

Erik V. Berg ${ }^{1 *}$, Heather J. Gomes ${ }^{1}$, Charlotte L. Conturie ${ }^{1}$ and Richard O. Wein ${ }^{1}$

Correspondence: eberg@tuftsmedicalcenter.org

'Department of Otolaryngology - Head and Neck Surgery, Tufts Medical Center, Tufts University School of Medicine, Boston, MA, USA.

\begin{abstract}
Background: We describe the management of a pregnant female with preeclampsia, who presented at 33 weeks gestation in respiratory distress secondary to compression from a multinodular goiter.

Results: A multidisciplinary team was assembled, including anesthesiology, maternal fetal medicine, otolaryngology, and cardiothoracic surgery. The patient underwent a cesarean section followed by a total thyroidectomy for removal of the compressive thyroid goiter. Cardiothoracic surgery was included in this particular case because of the risk for acute loss of the airway, which could have necessitated cardiopulmonary bypass.

Conclusions: Airway obstruction from extra-tracheal compression in the setting of preeclampsia represents a unique management dilemma. Successful results can occur with a multidisciplinary approach to airway management.
\end{abstract}

Key words: multinodular goiter, airway compression, pregnancy, preeclampsia, cardiopulmonary bypass

\section{Background}

Thyroid disease is relatively common among the general population, and certain thyroid conditions may be more common among pregnant women. Infrequently, thyroid conditions require surgical intervention during pregnancy. Such instances include failure of medical management of thyrotoxicosis, compromised vision secondary to Graves' orbitopathy, or compromised airway due to compression of the trachea [1].

Acute airway compromise coupled with a complicated pregnancy is exceedingly rare and requires complex management. A multispecialty team approach is needed for surgical procedures. We describe a pregnant patient, who presented to our institution in her third trimester, with a large neck mass and worsening respiratory symptoms.

\section{Methods}

A case report, at an academic medical center, that involves the complex management of an acute airway a pregnant patient in her third trimester.

\section{Case Report-Results}

A 31-year-old female presented at 33 weeks gestation complaining of severe headache. After assessment by obstetrics, she was diagnosed with preeclampsia. She also reported difficulty breathing when recumbent for 3 weeks. She had a 3-year history of a thyroid nodule, and previous fine-needle aspiration showed atypical cells suspicious for papillary carcinoma. She had no other significant medical or family history.

On examination, she was hypertensive and in no apparent distress, but she became short of breath and had biphasic stridor when supine. She had a large, midline neck mass obscuring the thyroid cartilage and sternal notch (figure 1). The mass was firm, non-mobile, non-tender, and measured $5 \mathrm{~cm}$. Bedside flexible fiberoptic laryngoscopy was normal. Laboratory tests showed normal thyroid function, electrolytes, and complete blood count. A CT with intravenous contrast of the neck and chest showed a markedly enlarged heterogeneous thyroid gland with substernal extension and compression of the trachea by greater than $50 \%$ of the baseline diameter (figures $2 \& 3$ ). No suspicious lymphadenopathy was noted and the findings were consistent with a multinodular goiter.

Based on the complexity of the case, a multidisciplinary team was assembled which included: Anesthesiology, Maternal Fetal Medicine, Cardiothoracic Surgery, and Otolaryngology. The multispecialty operative plan was planned to occur in three stages.

The first stage focused on keeping the mother comfortable without manipulation of the airway and delivering the infant. The mother would undergo a cesarean section due to severe preeclampsia at 33 weeks gestation. Epidural, with 2\% lidocaine, was placed between L4-5, and used for anesthesia. Her hypertension was controlled with IV labetolol as needed. The head of bed was elevated to keep the mother comfortable and avoid airway compression. The anesthesiologist placed a radial 


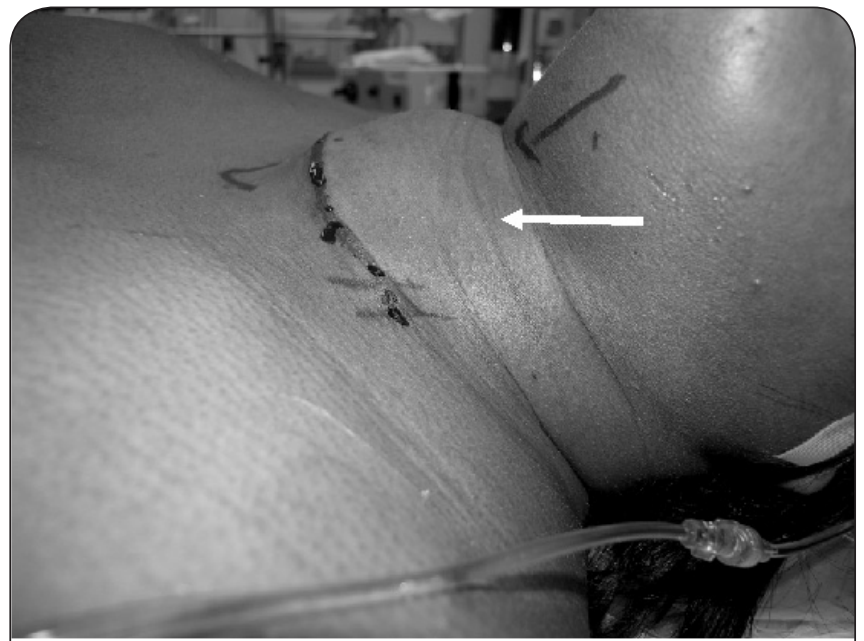

Figure 1. Preoperatively, showing compressive thyroid goiter (arrow).

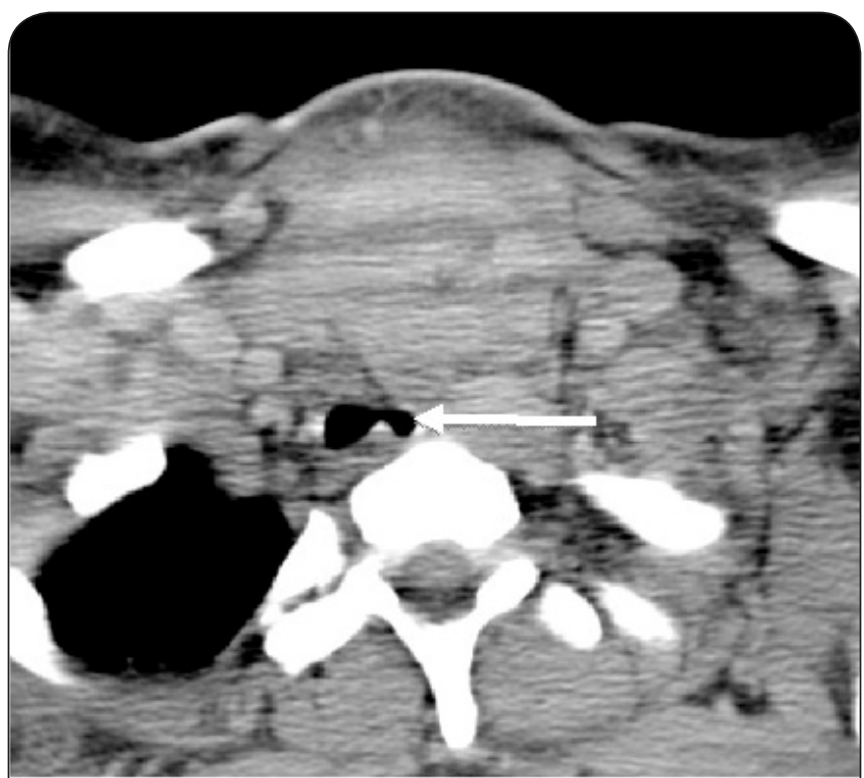

Figure 3. Axial soft tissue computed tomography (CT) showing mass effect on the trachea deviating it to the right (arrow).

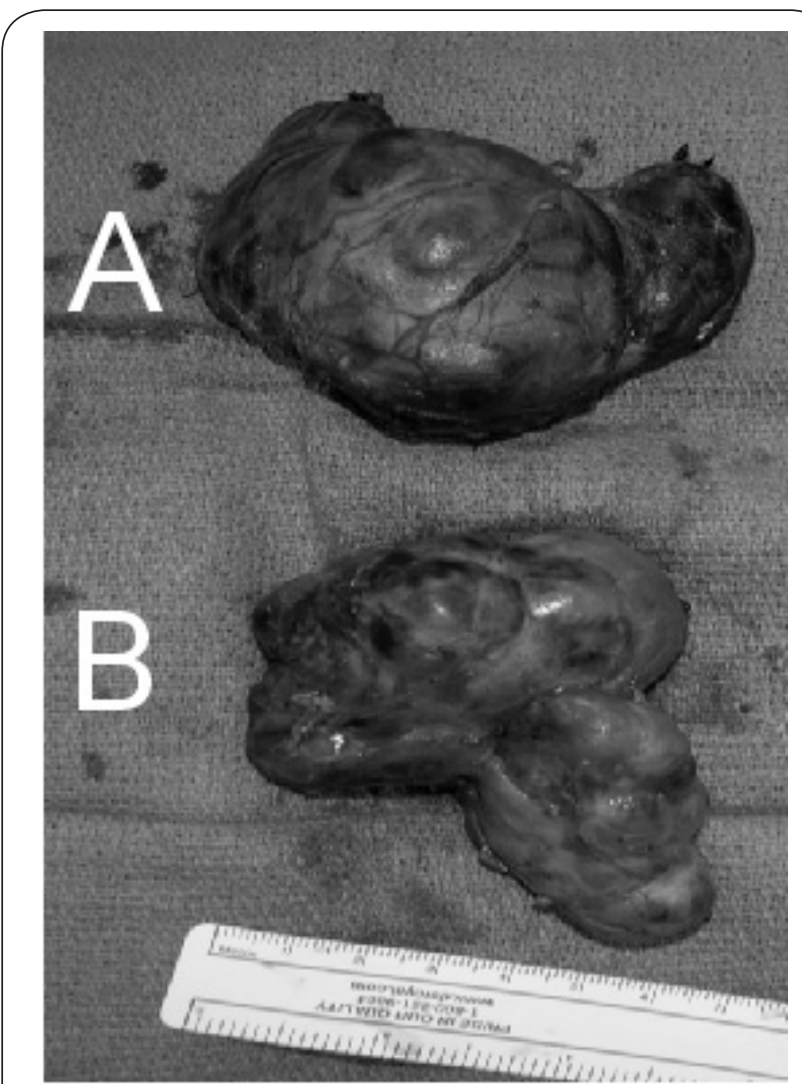

Figure 4: A. Thyroid Mass B. Substernal Component 
arterial line and left femoral central line. Prior to incision, the mother was treated with $3 \mathrm{~mL}$ of nebulized $4 \%$ lidocaine to topically anesthetize the airway. The Cardiothoracic Surgical team utilized the epidural anesthesia to prepare the right femoral vessels for potential cardiopulmonary bypass (CPB). The Obstetric team subsequently delivered a healthy infant, APGAR of 7 and 8, via cesarean section.

After delivery, the second stage focused on the mother's airway at induction of anesthesia and intubation. Given the concern for the potential loss of airway at induction, the intubation was planned to also proceed in a stepwise fashion with rigid bronchoscopy as alternative to stabilize the airway. The Anesthesiologist maintained the head of bed in an elevated position. An awake fiberoptic intubation was successful on the second attempt using a 6-0 ETT. The mother was initially treated with dexmedetomidine and fentanyl and then transitioned to isoflurane, fentanyl, and propofol for the remainder of the procedure.

The final stage of the plan involved the Otolaryngologist removing the multinodular substernal goiter. The incision was injected with $1 \%$ lidocaine and 1:100,000 epinephrine. Total thyroidectomy was performed with identification and preservation of the left inferior and right superior and inferior parathyroids. The avascular left superior parathyroid was identified on the left upper lobe and reimplanted in left sternocleidomastoid muscle. There was noted to be fibrous band between the main total thyroidectomy specimen and the goitrous substernal component that allowed for an avascular separation of the two components and the removal as separate specimens. (figure 4). Both recurrent laryngeal nerves were preserved and no significant lymphadenopathy was identified. The tracheal rings compressed by the substernal goiter were noted to be malacic. The patient remained intubated postoperatively and was transferred to the surgical ICU. She was extubated, after short course of steroids with audible air leak noted around ETT with cuff deflated, on postoperative day 2 and remained asymptomatic. She had transient postoperative hypocalcemia that resolved prior to discharge on postoperative day 7 . The final pathology was consistent with multinodular goiter with a solitary, subcentimeter, papillary micro-carcinoma.

\section{Discussion}

The literature contains several case studies that address the management of distal tracheal obstruction during pregnancy [3-4]. Cunningham in 1970 was the first case series in the literature [4]. His results show 3 fetal deaths out of five cases. More recently, Nam reported 6 cases of thyroidectomy during the second trimester with no increase in complications or poor outcomes for the fetus [5]. Kuy et al compared 165 pregnant women who underwent thyroidectomy to over 31,000 non-pregnant thyroidectomies [6]. They found a statistically significant difference in general complications in $11.4 \%$ of pregnant patients versus $3.6 \%$ in non-pregnant patients. The overall fetal complication rate was $5.5 \%$ and maternal complication rate was $4.5 \%$; however, there was no data on surgeries performed due to airway compromise. None of the literature regarding pregnant thyroid patients discussed multidisciplinary care or cardiopulmonary bypass as an option for loss of airway.

The first stage of the procedure, the cesarean section and vessel preparation, was performed under spinal anesthesia for two reasons. First, an attempt at intubation at the beginning of the case put two lives at risk, the mother and infant, if the airway was compromised. Performing a cesarean under regional anesthesia with the mother spontaneously breathing removed this risk. Second, with successful epidural anesthesia, the Cardiothoracic Surgical team, were able to prepare the right femoral vessels for CPB if the airway was lost.

The anesthesiologist had to account for the physiologic changes due to both pregnancy and preeclampsia. Pregnancy causes diaphragmatic compression by the uterus, decreased tone in the lower esophageal sphincter, and prolonged gastric emptying, which increases the risk of aspiration at intubation. In preeclampsia, increased edema and fluid retention can result in swelling in the airway and loss of landmarks during intubation.2 Thus, relatively minor trauma to the larynx could potentially result in complete airway loss.

Given these considerations, the plan for the airway was to perform an awake, fiberoptic intubation with topical lidocaine anesthesia. If unsuccessful, an attempt at jet ventilation would ensue followed by rigid bronchoscopy to regain control of the airway. Although tracheotomy was discussed, given the relative size of the goiter, this was considered a poor option. Lastly, the patient would be put on CPB if all previous steps failed

In the case presented, prepping for cardiopulmonary bypass was considered, but the evidence supporting it is mixed. Slinger found that it is not a realistic option [7]. Even with vessel preparation it would take a minimum of 5 to 10 minutes to start. This anoxic period would cause severe neurologic injury. Conversely, Goh et al. based on experience and a review of the literature, recommended having the bypass vessels prepped and ready for potential loss of airway in patients with greater than $50 \%$ obstruction of the trachea [8]. Our patient's CT scan showed greater than 50\% obstruction; therefore, CT surgery prepped the vessels and also felt, once ready, they could have bypass in place within a few minutes.

It should be noted that the risks of bypass are not benign. ${ }^{3}$ First, there is a risk of femoral vessel thrombosis leading to distal ischemia of the lower leg and foot. Second, heparin is used to prevent clotting in the tubing, making patients susceptible to CNS hemorrhage. Lastly, there is a risk of renal and/or equipment failure. Given these risks, the multidisciplinary team felt the potential benefit in an airway emergency outweighed the risks of bypass.

Our patient had a good outcome due to effective planning. The time spent discussing options with a multidisciplinary 
team was critical to preventing untoward outcomes. Every team member offered different expertise and surgical skills; however, knowing when to use the right tools was critical, especially in the management of the airway. Effective communication among team members allowed for development of a comprehensive plan prior to entering the operating room.

\section{Conclusion}

Acute airway obstruction in the setting of a complex pregnancy is a unique management dilemma. A multidisciplinary approach and careful planning was integral to the appropriate management of this patient. The members of the team are determined by the complexity of the case. Cardiopulmonary bypass is a potentially lifesaving airway management option in selective cases. Preparing for potential difficulties and communicating effectively can lead to successful results and avoid catastrophic outcomes.

\section{Competing interests}

The authors declare that they have no competing interests.

\section{Authors Contributions}

EB. Majority of Background, Discussion/Conclusions, Editing HG. Minority of Background, Discussion/Conclusions, Editing CC. Details of Case Report/Results, Minor Editing RW. Supervision and Minor Editing

\section{Publication history}

Received: 21-May-2012 Revised: 10-Aug-2012

Accepted: 23-Aug-2012 Published: 28-Aug-2012

\section{References}

1. Preston TW, Lee WJ, Stack BC, Jr.: Report of an obstructive goiter and its surgical treatment during delivery. Am J Otolaryngol 2004, 25(5):364-7. | Article | PubMed

2. Munnur $U$, de Boisblanc $B$, Suresh MS: Airway problems in pregnancy. Crit Care Med 2005, 33(10 Suppl):S259-68. | Article I PubMed

3. Szokol JW, Alspach D, Mehta MK, Parilla BV, Liptay MJ: Intermittent airway obstruction and superior vena cava syndrome in a patient with an undiagnosed mediastinal mass after cesarean delivery. Anesth Analg 2003, 97(3):883-4. | Article | PubMed

4. Cunningham MP, Slaughter DP: Surgical treatment of disease of the thyroid gland in pregnancy. Surg Gynecol Obstet 1970, 131(3):486-8. I PubMed

5. Nam KH, Yoon JH, Chang HS, Park CS: Optimal timing of surgery in well-differentiated thyroid carcinoma detected during pregnancy. J Surg Oncol 2005, 91(3):199-203. | Article | PubMed

6. Kuy S, Roman SA, Desai R, Sosa JA: Outcomes following thyroid and parathyroid surgery in pregnant women. Arch Surg 2009, 144(5):399-406; discussion. | Article | PubMed

7. Slinger $P$, Karsli C: Management of the patient with a large anterior mediastinal mass: recurring myths. Curr Opin Anaesthesiol 2007, 20(1):1-3. | Article | PubMed

8. Goh MH, Liu XY, Goh YS: Anterior mediastinal masses: an anaesthetic challenge. Anaesthesia 1999, 54(7):670-4. | Article | PubMed 